Int. J. Speleol. 10 (1978), pp. 35 - 55

\title{
Réponses locomotrices du poisson cavernicole Astyanax jordani (Pisces, Characidae) à des signaux périodiques et apériodiques de lumière et de température.
}

\author{
par
}

\section{G. THINES ET M. WEYERS}

Locomotor responses of the cave fish Astyanax jordani (Pisces, Characidae) to periodic and aperiodic light and temperature signals.

\section{SUMMARY}

The locomotory activity of adult cave fishes Astyanax jordani was recorded in isolation in the following light and temperature conditions: constant conditions $\left(100 \mathrm{Lx}-20^{\circ} \mathrm{C}\right)$, in a light cycle (LD:11/11 - $10 \mathrm{Lx}-100 \mathrm{Lx})$ and in a temperature cycle $\left(11 / 11 ; 17 / 20,19 / 22,20 / 23,27 / 30^{\circ} \mathrm{C}\right)$. All longitudinal time series extended for a minimun of 30 days.

Results show: (1) That no circadian regulation appears in constant conditions; (2) that passive entrainment occurs in LD (Amplitude: $90 \mathrm{Lx}$ ) and in periodic temperature conditions (Amplitude: $3^{\circ} \mathrm{C}$ ). The entrainment effect damps out and varies individually; (3) that the mean activity increases with temperature; (4) The adjustment of activity to periodic signals is individually stable. These results suggest that $A$. Jordani is devoid of any endogenous oscillator of the circadian type. The observed thermal adaptation could have the following functions: (1) To increase the level of activity in function of the thermal level under the form of passive entrainment; (2) To enhance the exploratory behaviour of the fish in search of a termal preference allowing the animal to keep inside a well defined zone of the subterranean biotope in relation to small local temperature changes.

\section{INTRODUCTION}

Le problème de l'existence éventuelle de rythmes biologiques chez les Poissons cavernicoles relève à la fois de l'évolution régressive de ces formes et des faits relatifs aux rythmes biologiques des Poissons épigés susceptibles d'éclairer le problème tel qu'il se pose chez les formes régressées. Une hypothèse d'ensem-

\footnotetext{
${ }^{(1)}$ Centre de Psychologie expérimentale et comparée, Université de Louvain, B - 3041 Pellenberg (Belgique).
} 
ble pourrait être formulée, selon laquelle la dégénérescense phylétique des Poissons cavernicoles aurait affecté les régulations temporelles de la forme ancestrale en raison de l'utilité biologique faible, voire nulle, que celles-ci présentent théoriquement dans le milieu souterrain, lequel est largement soustrait à la plupart des influences cycliques du milieu épigé. Cependant la constance du milieu aquatique souterrain ne saurait être affirmée sans réserves. Elle est évidente en ce qui concerne l'obscurité dans la zone profonde d'un système karstique et exclut de ce fait tout synchroniseur lumineux capable de régler d'éventuelles variations circadiennes d'activité chez ces espèces. C'est semblet-il également le cas pour les variations thermiques dans ces même zones pour des valeurs cycliques de l'ordre du nycthémère. Par contre, cette constance semble moins évidente sur le plan saisonnier, particulièrement dans les grottes tropicales, qui sont en outre les seules à abriter des Poissons cavernicoles (Thinès, 1969). On sait en particulier que d'importantes modifications annuelles surviennent dans ces biotopes en lien avec le cycle des pluies et que l'inondation saisonnière des grottes accroît périodiquement les réserves nutritives souterraines. Divers signaux chimiques et thermiques interviennent à ce moment dans la régulation du cycle vital de certain poissons cavernicoles (Heuts, 1951, 1953) et particulièrement dans le déclenchement de la reproduction. On doit à Husson (1971) une synthèse critique des principaux faits connus au sujet des rythmes biologiques des cavernicoles. Les conclusions de cet auteur, dont l'enquête porte sur un matériel étendu au point de vue taxonomique, soulignent l'importance des variations périodiques dans la composition de la faune souterraine ansi que des rythmes saisonniers, mais insistent par ailleurs sur l'absence de rythmes circadiens chez les troglobies, à quelques rares exceptions près. Ces faits rendent très plausible l'hypothèse que le système circadien endogène des cavernicoles caractérisés aurait subi une dégénérescence phylétique comparable à celle qu'ont subie d'autres systèmes comportementaux. Celle-ci est bien établie chez les Poissons cavernicoles en ce qui concerne la tendance grégaire des Cypriniformes (Caecobarbus geertsi, Typhlogarra widdowsoni et Anoptichthys jordani; Thinès, 1969) et la réponse à la substance d'alarme chez les Characidae et certains Cyprinidae cavernicoles (Pfeiffer, 1963-1966 -Thinès et Legrain, 1973). La structure du comportement alimentaire a subi de son côté des transformations dégénératives, accompagnées toutefois de certaines substitutions constructives dans la polarisation spatiale de l'exploration, chez Caecobarbus et chez Anoptichthys (Thinès, Soffié et Vandenbussche, 1967 - Thinès et Wissocq, 1973 - Thinès et Capon, 1975).

Des observations récentes sur le Poisson cavernicole Lucifuga subterranea (Ophidiidae) suggèrent toutefois que les conséquences de la dégénérescence phylétique peuvent se traduire par des modifications structurales très différentes dans les réponses à diverses stimulations (Thinès et Piquemal, 1978). L'important est, à notre sens, que des dégénérescences phylétiques aient été mises en évidence au niveau des comportements eux - mêmes; ce fait permet d'aborder en principe l'étude de la structure des conduites temporelles des Poissons cavernicoles dans une perspective du même ordre. 
L'étude des caractères dégénératifs des cavernicoles dans la perspective de la génétique, telle qu'elle a été menée par Kosswig (1962, 1963, 1965), Parzefall (1973), G. Peters (1973), N. Peters et G. Peters (1973), Schemmel (1973), Wilkens (1973) et d'autres encore, repose essentiellement sur l'idée que la pression mutatoire n'est plus contrôlée à un taux significatif par la pression sélective dans les biotopes souterrains, en sorte que les modifications des caractères seraient presque exclusivement déterminées par la pression mutatoire. En outre les caractères dénués de signification biologique et les caractères neutres pourraient s'être maintenus par l'action de gènes pléiotropiques. C'est avant tout l'application de cette théorie aux conduites temporelles qui mène à l'hypothèse déjà mentionnée selon laquelle certains mécanismes régulateurs des rythmes biologiques ancestraux auraient dégénéré par suite de leur faible utilité biologique dans des milieux privés de certains synchroniseurs. En outre, certains autres régulateurs rythmiques endogènes pourraient s'être maintenus par l'effet de la pléiotropie. Il n'est donc pas exclu à priori que des rythmes endogènes aient pu persister chez les cavernicoles en raison de la valeur adaptative de leur période propre dans le contexte du milieu souterrain, tandis que d'autres auraient dégénéré pour la raison inverse.

Ceci fournit en première approximation un critère susceptible d'expliquer l'absence quasi génerale de rythmes circadiens et la persistance marquée de rythmes circanniens relevée par Husson (1971) chez des formes cavernicoles très diverses.

D'autres considérations théoriques développées par Heuts (1951) et discutées par Thinès (1969) définissent, à notre avis, une dimension fondamentale supplémentaire dans l'analyse de la signification biologique possible des conduites temporelles des cavernicoles. La théorie de Heuts (1951-1953) élaborée à partir des travaux de cet auteur sur le Cyprinidae cavernicole Caecobarbus geertsi, souligne particulièrement l'abaissement spécifique du taux métabolique de base et le ralentissement considérable du développement ontogénétique de cette espèce par rapport aux formes épigées les plus proches. Heuts interprète ces deux faits comme deux indices de maximalisation de l'état troglobiotique manifestant une déficience des régulations internes, cette dernière permettant aux régulations externes du milieu d'imposer aux cavernicoles leur rythme de vie au ralenti. Cette passivité caractéristique des organismes cavernicoles, qui correspond très précisément à l'inféodation sans cesse rappelée des troglobies au milieu souterrain, amène elle aussi à supposer que l'autonomie typique d'un système circadien ancestral (hypothétique) pourrait avoir été atteinte au cours de ce processus. On comprendrait sans peine, de ce point de vue encore, l'absence des rythmes circadiens chez les cavernicoles vrais et chez les Téléostomes en particulier.

Outre les faits de l'évolution régressive qui viennent d'être mentionnés, il y a lieu d'envisager brièvement la question des rythmes biologiques chez les Téléostomes en général. C'est en effet sur cette base qu'il convient d'évaluer les conduites temporelles des Poissons cavernicoles, lesquels ont tous évolué à partir de formes épigées. L'examen des principaux travaux consacrés à cette 
question révèle quelques faits fondamentaux qu'il importe d'évaluer critiquement. Dans la révision d'ensemble qu'il a consacrée à ce problème, Schwassmann (1971) estime que chez la plupart des Téléostomes étudiés à cette date, les indications sur les rythmes circadiens se limitent à mettre en évidence la p e r s i s -

tance de certaines fonctions périodiques dans des conditions constantes. Lissmann et Schwassmann (1965) avaient d'ailleurs montré que les décharges périodiques des organes électriques de Gymnorhamphichthys hypostomus présentaient une périodicité circadienne. En ce qui concerne l'existence d'un rythme typique de l'activité locomotrice, Siegmund et Wolff (1973) estiment qu'il existe chez Leucaspius delineatus et qu'il présente chez cette espèce la capacité de synchronisation caractéristique de rythmes circadiens authentiques. Il est un fait que plusieurs Téléostomes dulcaquicoles présentent des distributions d'activité typiques en conditions constantes (Harrington, 1963, Thinès, 1970, Stickney, 1972, Andreasson, 1973, Varanelli et Mc Cleave, 1974). Cependant, tout en mettant en évidence certains phénomènes d'entraînement, ces données suggèrent que la stabilité de la période est en général moindre chez les Poissons que chez certains Invertébrés et certains Vertébrés terrestres (Aschoff, 1965, Gibson, 1967, Hoffmann, 1969, Medioni, 1967, Palmer, 1974, Weyers, 1974, Rensing, 1972). L'étude des rythmes biologiques des Téléostomes épigés ne permet donc pas, dans l'état actuel des choses, de supposer que les formes épigées ancestrales des Poissons cavernicoles auraient manifesté des rythmes biologiques endogènes au niveau du comportement plus caractéristiques que ceux des espèces actuellement étudiées. L'hypothèse d'une évolution dégénérative d'un tel mécanisme de «copie» d'un système régulateur endogène au niveau du comportement, telle qu'elle est mentionnée plus haut est donc faiblement étayée au premier examen. Par contre, l'hypothèse d'un entraînement passif par les rythmes exogènes, principalement saisonniers, semble plus conforme aux faits d'ensemble relevés sur les espèces épigées précitées et cadre en outre mieux avec les conditions écologiques qui caractérisent le milieu aquatique souterrain.

Nous avons procédé antérieurement (Thinès et all., 1965) à une étude préliminaire sur la répartition temporelle de l'activité locomotrice de la forme épigée et de la forme cavernicole d'Astyanax mexicanus ainsi que sur les hybrides $\mathrm{F}_{1}$ et $F_{2}$ issus du croisement de ces deux formes. La forme épigée présente des distributions typiques d'activité en réponse à des signaux lumineux périodiques, mais les distributions restent apériodiques en conditions constantes, tant chez la forme épigée que chez la forme cavernicole. Au vu de ces résultats, on pouvait se demander si l'absence d'un rythme caractérisé chez l'Astyanax, tant épigé que cavernicole, n'était pas imputable à la nature des signaux utilisés autant qu'à une déficience de principe de l'oscillateur endogène lui-même. On sait en effet, que le rythme auto-entretenu en conditions constantes n'apparaît souvent que pour une bande étroite de l'échelle d'amplitude du signal constant (Winfree, 1974) et que souvent cette valeur efficace est sans rapport avec le niveau moyen observé dans le milieu naturel. C'est pour cette raison que dans la 
présente recherche, nous avons utilisé à la fois des signaux lumineux et des signaux thermiques, en vue d'établir si la combinaison de ceux-ci ne fournirait pas de réponses plus typiques.

\section{Matériel et méthodes}

Etant donné les restrictions mentionnées ci-dessus au sujet de la stabilité des rythmes biologiques chez les Téléostomes étudiés à ce jour et compte tenu du fait que les rythmes circadiens peuvent présenter une variabilité intraspécifique et un polymorphisme inter-spécifique accusés (Saint Girons, 1971, Müller, 1973, a, b, Hoffmann, 1969, 1973), il était difficile de décider d'un schéma expérimental satisfaisant sous tous les rapports. Notre choix méthodologique s'est finalement porté sur un plan longitudinal prolongé dans lequel un nombre limité d'individus fut soumis à un programme comprenant diverses conditions successives périodiques ou constantes tant du point de vue de l'éclairement que du point de vue de la température. Trois individus adultes du genre Astyanax forme cavernicole ${ }^{(1)}$ ayant séjourné 18 mois dans des conditions constantes $\left(22^{\circ} \mathrm{C}, 100 \mathrm{Lx}\right)$ avant le début de l'expérience furent placés pour cette raison dans un dispositif isolant permettant des combinaisons variées des deux types de signaux (Weyers, 1975), l'ensemble des expériences effectivement réalisées s'étalant sur un total de 16 mois (fig. 1). La figure 1 reprend quelques séquences réprésentatives des diverses conditions imposées qui portent sur $50 \%$ de la durée totale de l'expérience. Bien que les Poissons aient été soumis sans interruption à la séquence de conditions représentées à la fig. 1, l'activité locomotrice n'a pas été enregistrée de manière continue durant les 16 mois de l'expérience, les intervalles d'adaptation ou les quelques jours consécutifs à la distribution de nourriture vivante étant négligés.

Les Poissons étaient isolés individuellement dans des compartiments à double paroi et les aquariums étaient protégés des vibrations par des matériaux absorbants de différentes densités. Les liaisons électriques et tubulaires étaient conçues de manière à établir des connexions souples entre les aquariums et les installations de contrôle des eaux et d'enregistrement. Le sous-sol dans lequel se trouvaient les compartiments étaient relativement bien isolé dans le bâtiment et les bruits continus de la pompe de circulation et de la pompe à air exerçaient un effet masquant complémentaire. Les compartiments étaient reliés à un réservoir commun comprenant un filtre, un élément refroidisseur et une résistance chauffante. Le réservoir était alimenté de façon continue en eau et en air frais de sorte que le milieu aquatique expérimental était constant sous le

(1)Le genre Anoptichthys n'étant plus admis, l'ancienne dénomination Anoptichthys jordani doit être remplacée par Astyanax jordani pour qualifier la forme cavernicole de l'Astyanax mexicanus (cf. Sadoglu, 1957) 
40

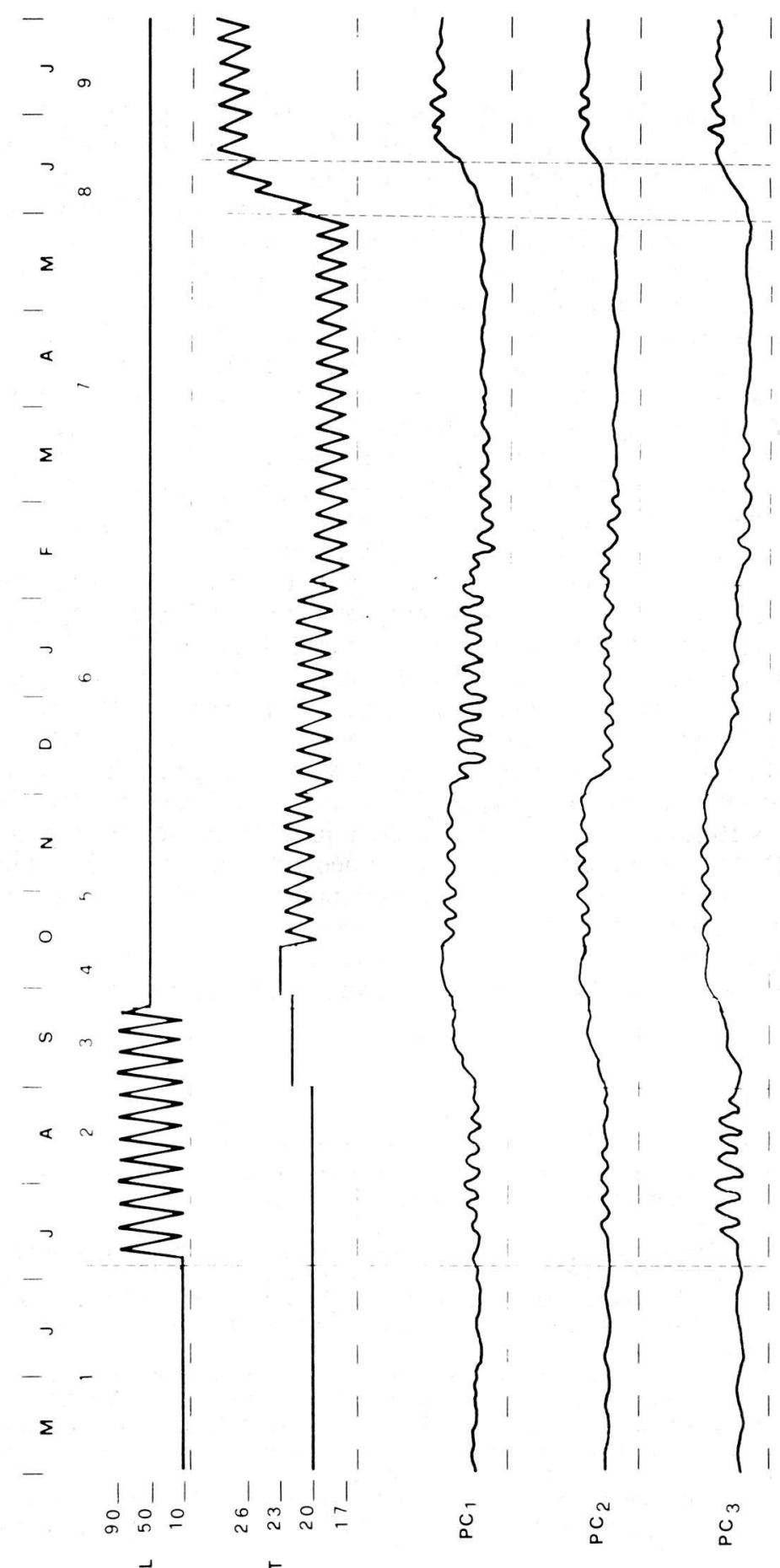


rapport de l'oxygénation (100\%) et de la turbidité. La circulation de l'eau était assurée par une pompe et le débit de chaque aquarium était semblable. Chaque aquarium était surmonté d'une source lumineuse constituée de 4 ampoules à incandescence commutables indépendamment. L'éclairement, dans l'aquarium, pouvait aller de 0 à $150 \mathrm{Lx}$ selon le nombre de lampes en fonctionnement et leur position dans le tube. La température de l'eau était mesurée par un thermostat électronique à la sortie de la pompe $\left(1 / 5 \mathrm{de}{ }^{\circ} \mathrm{C}\right.$ de précision) et la température des aquariums était enregistrée après conversion en valeurs digitales. L'activité locomotrice des Poissons était détectée par l'interruption de deux faisceaux parallèles de lumière infra-rouge traversant l'eau à $3 \mathrm{~cm}$ du fond et $4 \mathrm{~cm}$ des parois verticales latérales. Des phototransistors transformaient les interruptions des faisceaux en impulsions qui étaient accumulées dans les compteurs pendant une heure (intégration horaire). Les séquences temporelles étaient donc constituées de 24 valeurs horaires par jour, pour les 3 aquariums et pour la température et l'éclairement.

Les signaux utilisés sont résumés à la fig. 1 . Les niveaux constants d'éclairement ont été de 10 ou de $50 \mathrm{Lx}$ et ceux de la température de $20^{\circ}, 22^{\circ}$ et $23^{\circ} \mathrm{C}$. Les signaux périodiques ont toujours été de $22 \mathrm{~h}$ de période $(11$ heures maximum, 11 heures minimum, afin d'isoler les effets induits par les signaux imposés d'éventuels effets de signaux parasites de $24 \mathrm{~h}$ de période) avec une amplitude de $80 \mathrm{Lx}(10 / 90)$ pour l'éclairement et de $3^{\circ} \mathrm{C}\left(17^{\circ} / 20^{\circ}, 19^{\circ} / 23^{\circ}\right.$, $27^{\circ} / 30^{\circ}$ ). En ce qui concerne l'alimentation des Poissons, de la nourriture sèche était introduite en petite quantité à des intervalles d'au moins 4 jours et d'au plus 10 jours et à des moments de la journée qui variaient systématiquement. De la nourriture vivante était donnée à des intervalles également variables mais beaucoup plus espacés ( 30 à 60 jours) et le plus souvent une semaine avant une modification des conditions imposées.

En ce qui concerne le traitement mathématique des données, la préférence a été accordée à la méthode du profilogramme développée par Citta (1977).

Fig. 1. - Réprésentation schématique des conditions imposées de température et d'éclairement et des réponses locomotrices de 3 Astyanax jordani.

La première et la deuxième ligne résument les conditions d'éclairement $(\mathrm{L})$ et de température $(\mathrm{T})$ : une ligne horizontale continue représente des conditions constantes et une dent de scie représente des conditions périodiques (signal symétrique de forme carrée $-11 \mathrm{~h} / 11 \mathrm{~h}$ - de période $=22 \mathrm{~h}$ ). Le niveau d'éclairement est indiqué en Lx (mesuré dans l'eau des aquariums) et celui de la température en degrés $\mathrm{C}$.

Les trois lignes suivantes représentent l'évolution des réponses des Poissons. Le niveau d'activité est représenté par rapport à la moyenne réduite des 15 mois d'expérience. Les valeurs sont calculées sur des sous-séquences de 120 heures décalées de 24 heures (chevauchement). Lorsque les réponses présentent une structure périodique, c'est-à-dire lorsque la valeur chi-carré correspondant à $22 \mathrm{~h}$ dépasse le seuil de 1.2 , la ligne figurant les réponses est modulée par une oscillation d'allure sinusoîdale dont l'amplitude est proportionelle à la grandeur du chi-carré. Les séries successives sont indiquées par les chiffres de 1 à 9 , les mois par la première lettre du mois correspondant. 

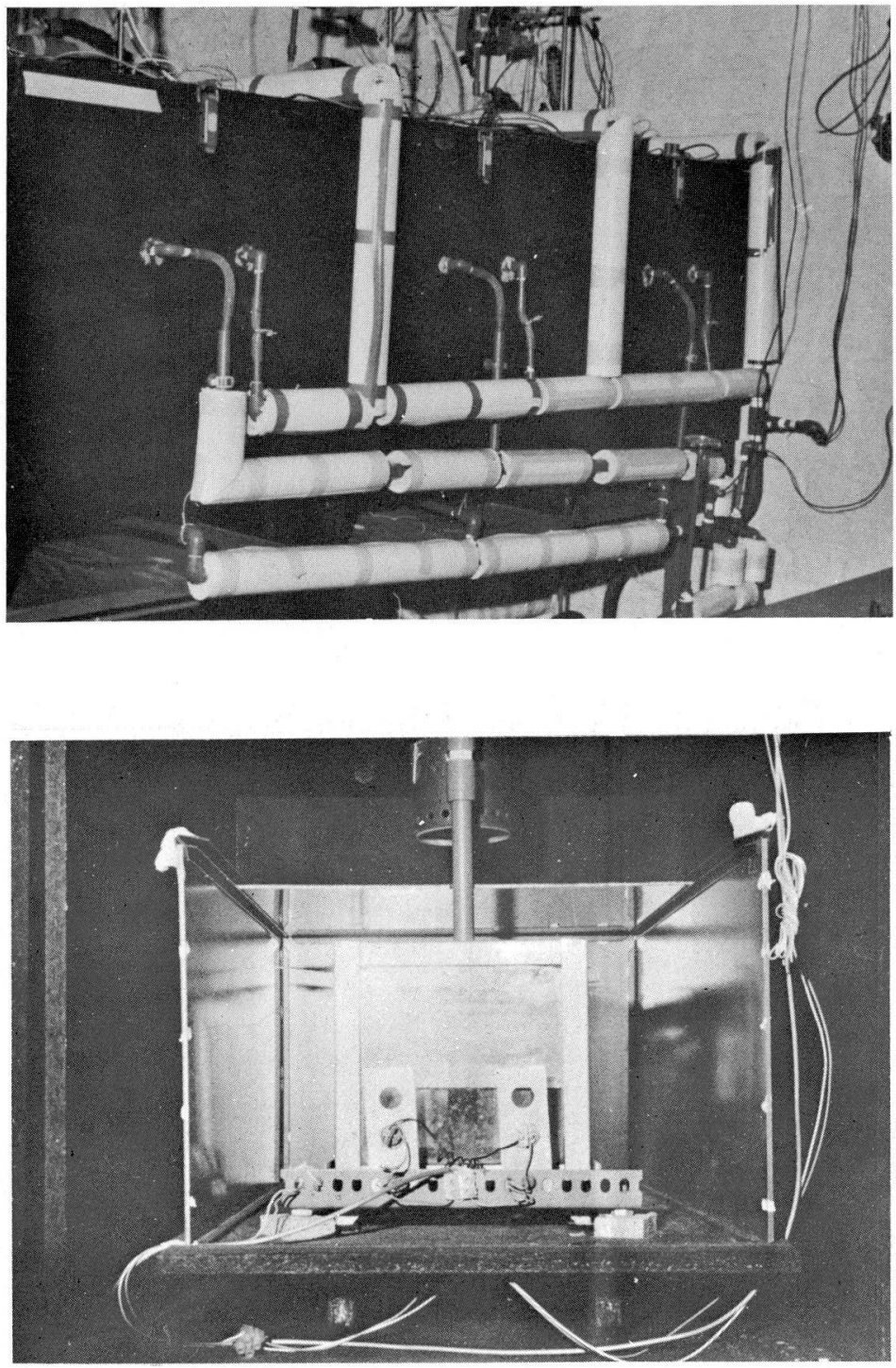

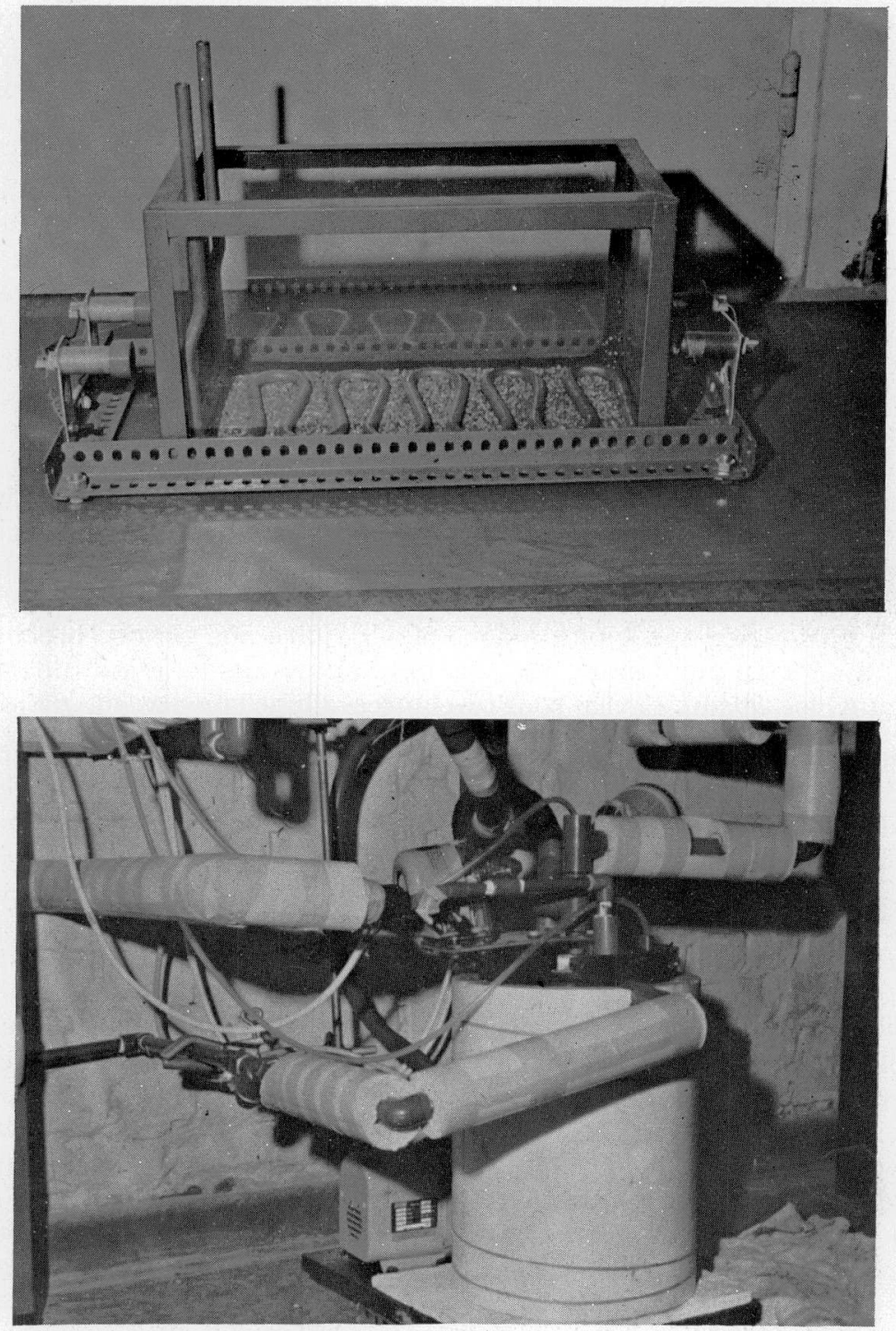

Planche 1.

Dispositif expérimental utilisé dans les expériences sur les réponses locomotrices d'Astyanax jordani: (a) Vue générale des compartiments d'isolement; (b) Vue frontale d'un aquarium expérimental à l'intérieur d'un compartiment; (c) Vue latérale d'un aquarium expérimental montrant les détecteurs photoélectriques et les éléments chauffants; (d) Pompe de circulation et filtre. 
Dans la mesure où il s'agissait de détecter des réponses périodiques, qu'elles soient d'origine endogène ou exogène, et compte tenu du fait que les données relatives au comportement locomoteur des Poissons et à ses régulations temporelles semblent sujettes à de fortes variations, cette méthode semblait la plus pertinente. Les méthodes classiques, l'auto-corrélation et l'analyse de Fourier en particulier, sont moins indiquées vu la nature des données recueillies. Le caractère stationnaire des séries n'était pas établi au départ et les modulations de fréquence étaient hautement probables, de sorte que l'application de ces méthodes à des sous-séquences courtes ou à des séries à forte dérive aurait rendu leur interprétation moins légitime.

La méthode du profilogramme dont on trouvera un exposé complet dans l'article précité de Citta (1977) est en bref la suivante: Disposant d'une séquence temporelle de $\mathrm{N}$ valeurs équidistantes, il est possible de calculer, pour toutes les périodes désirées (depuis $\mathrm{t}_{1}>2$ jusqu'à $\mathrm{t}_{\mathrm{n}} \leqslant \mathrm{N} / 2$ ) les profils qui expliquent une part de la variance totale de la séquence complète. En d'autres termes, la structure de la composante périodique de période $t_{j}$ est aussi bien décrite que possible par la série des moyennes de toutes les valeurs situées à une distance $t_{j}$ dans la séquence complète. La variance d'un tel profil des moyennes V (t) est d'autant plus élevée que le phénomène périodique a une période proche de $t_{j}$. Le calcul de V (t) pour toutes les périodes désirées donne le profilogramme, dans lequel les valeurs élevées sont révélatrices d'une composante périodique.

L'inconvénient de la méthode du profilogramme résulte de la dépendance des valeurs de V (t), soit lorsque ces valeurs sont adjacentes, soit lorsqu'elles sont en relation harmonique. En pratique toutefois, et, vu que la méthode présente l'avantage d'être applicable à de très courtes séries, elle peut être utilisée pour la détection de composantes périodiques si l'on tient compte de la possibilité qu'une valeur de $\mathrm{V}$ (t) élevée soit partiellement due à une composante de $\mathrm{V}$ $(\mathrm{t} / 2)$, cette dernière étant nécessairement due à une oscillation. Afin d'éviter des erreurs d'interprétation, les profilogrammes ont toujours été calculés pour des périodes allant de $5 \mathrm{~h}$ à $50 \mathrm{~h}$ afin d'y inclure les premiers multiples et sousmultiples de la période imposée (11 et 44 par rapport à 22). Comme les comparaisons présentées dans les figures ont été calculées avec les même paramètres de calcul (profilogrammes calculés sur des sous-séquences de 120 valeurs consécutives) il était possible de standardiser les résultats en les exprimant à travers une statistique chi-carré, comparant la variance des moyennes $V(t)$ à la variance théorique de la moyenne pour le même nombre d'observations. Les nombreuses applications antérieures de cette procédure sur des séquences réelles ou fictives ont montré qu'un dépassement du seuil 1.2 de la valeur chicarré, pour $\mathrm{N}=120$, impliquait la présence d'une oscillation. Le tableau 1 rapporte les résultats analysés selon cette procédure. Il en est de même pour la figure schématique 1 .

Avant l'analyse par le profilogramme, toutes les séquences on été filtrées par moyenne mobile $\left(y_{i}=a \cdot x_{i-1}+b \cdot x_{i}+a \cdot x_{i+1}\right.$, avec $a+b+a=1$ et $\left.b=0.5\right)$ qui n'affecte que les périodes inférieures à 5 , et réduites à une moyenne nulle et un écart-type $=1$. 


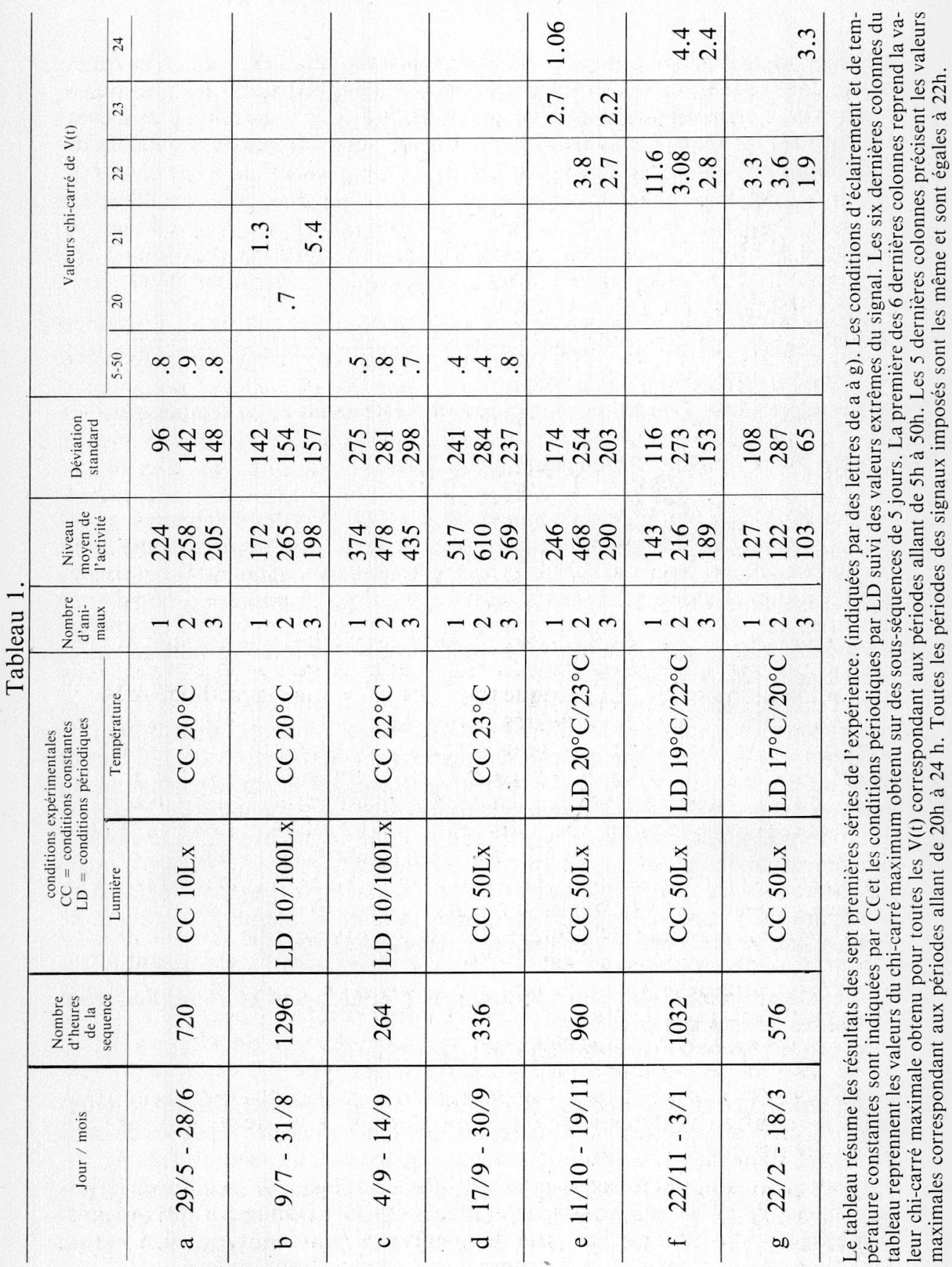


Les séquences d'activité et celles de la température n'ont été analysées qu'en partie dans le tableau I. Ce tableau reprend les sous-séquences les plus représentatives, c'est-à-dire celles qui présentent les indices chi-carré les plus élevés. Par contre, la figure 1 résume l'analyse complète de toutes les séquences disponibles.

\section{RESULTATS}

Les résultats d'ensemble sont représentés au tableau 1 et à la fig. 1 . L'examen de ces données permet de relever un certain nombre de phénomènes caractéristiques que nous analysons ci-après.

\section{1) Première série: Conditions constantes d'éclairement et de température}

$$
\text { (LL } 10 \mathrm{Lx}-\mathrm{t}: 20^{\circ} \mathrm{C} \text { ) (tableau 1,a fig. 1,1) }
$$

Au cours de cette première série qui couvre au total plus de 1400 heures, aucune activité périodique spontanée ne se manifeste. L'activité traduit quelques oscillations non typiques et variables ne présentant aucune persistance (plus de 2 ou 3 jours). Le niveau moyen d'activité, exprimé en nombre d'impulsions horaires, est du même ordre de grandeur chez chacun des trois Poissons en considérant la séquence entière de 1400 heures, mais la variabilité intraindividuelle reste élevée. En effet, des épisodes d'activité élevée se présentent chez l'un ou l'autre poisson sans qu'une covariation temporelle puisse être mise en évidence, exception faite pour les heures qui suivent la distribution de nourriture. Les valeurs de chi-carré, calculées sur des sections de 120 heures avec un décalage de 22 ou de 24 heures, ne révèlent aucun dépassement du seuil de 1.2 et confirment l'absence de toute expression locomotrice d'une régulation périodique interne de type circadien. Il n'est pas exclu que la variabilité génétique des poissons cavernicoles, soulignée par Kosswig (1965), intervienne dans la fréquence spontanée de l'activité locomotrice, mais même si elle apparaissait dans cet échantillon limité, le schéma longitudinal adopté ne permet pas de procéder à une analyse poussée.

\section{2) Deuxième série: Conditions périodiques d'éclairement et conditions con- stantes de température \\ (LD $11 / 11,10 / 90 \mathrm{Lx}, \mathrm{t}^{\circ} 20^{\circ} \mathrm{C}$ ) (tableau $1, \mathrm{~b}$ et fig. 1,2 ).}

Cette série couvre une durée de 1300 heures. Le signal périodique utilisé détermine un effet d'entraînement de la répartition temporelle des réponses de deux poissons, lesquelles atteignent des valeurs supérieures au seuil de 1.2 (fig. 1). Bien que ce ne soit pas le cas pour les trois Poissons et que la stabilité de l'ajustement au signal ne soit pas complète, ces résultats établissent qu'un signal carré de lumière entraîne l'activité de manière périodique. Le niveau moyen d'activité pour cet épisode reste comparable à celui du précédent. 
3) Troisième série: Les conditions sont semblables à celles de la deuxième série, mais le niveau de température est porté à $22^{\circ}$

(tableau 1,c; fig. 1.3).

Durant cet intervalle de 500 heures, dont 264 sont analysées à la fig. 3, l'effet d'entraînement disparaît à travers un accroissement du niveau moyen d'activité.

Le résultats des 3 premières séries indiquent donc:

1) un effet d'entraînement passif par les signaux lumineux cycliques;

2) un net accroissement de l'activité en fonction de l'élévation de la température;

3) un amortissement de la réponse périodique aux signaux lumineux parallèlement aux modifications des conditions thermiques.

4) Quatrième série: le signal d'éclairement est remplacé par des conditions constantes de lumière et le niveau de température est augmenté de $1^{\circ} \mathrm{C}$ (LL $50 \mathrm{Lx}, \mathrm{t} 23^{\circ} \mathrm{C}$, tableau 1,d; fig. 1.4)

Cet intervalle de 336 heures donne les même résultats que dans la première série sauf que le niveau moyen d'activité est plus élevé.

5) Cinquième série: A partir de cette série, toutes les séquences sont obtenues dans des conditions constantes d'éclairement (LL 50Lx) et sous des conditions périodiques de température dont l'amplitude est toujours de $3^{\circ} \mathrm{C}$ et la période de 22 heures (11/11). Dans cette cinquième série, les limites de variation du signal thermique sont de $20^{\circ} \mathrm{C}$ et de $23^{\circ} \mathrm{C}$ (tableau 1,e; fig, 1.5). L'épisode couvre une étendue de 1080 heures.

Les réponses périodiques des Poissons réapparaissent, avec les mêmes caractéristiques générales que celles qui sont obtenues sous le seul signal d'éclairement. Il y a donc dépassement de seuil 1.2 du chi-carré et instabilité relative de la réponse périodique associée à une dissociation interindividuelle des réponses. Deux poissons tendent à présenter un ajustement à la période du signal, mais on peut observer une composante différente pour l'un d'entre eux et pour le troisième. Vers la fin de l'épisode, les valeurs chi-carré tendent à redescendre au-dessous du seuil conventionnel.

Le niveau moyen des réponses, calculé sur l'ensemble de l'épisode, reste sensiblement voisin de celui qu'il avait atteint dans la série précédente. La variabilité est cependant élevée et est due essentiellement à des séquences (de quelques jours le plus souvent), dans lesquelles l'activité croît ou décroît sans qu'il soit possible de mettre en évidence une influence commune, exception faite pour les valeurs consécutives à une distribution de nourriture. 
Sixième série: seul le niveau de la température est modifié et le cycle thermique de cet épisode de 1560 heures est fixé entre les limites de $19^{\circ} \mathrm{C}$ et $22^{\circ} \mathrm{C}$. (tableau 1,f; fig. 1,6).

C'est dans cette série que l'on trouve les valeurs chi-carré les plus élevées. L'entraînement par la période 22 du signal thermique est très puissant et presque stable pour le premier poisson pour la totalité de l'épisode. Les deux autres manifestent l'intervention d'une composante de 24 heures s'ajoutant à celle qui correspond au signal. Chez ces derniers, et principalement chez le troisième, l'effet d'amortissement est presque total à partir de la moitié de la séquence.

La fig. 2-A présente le profil moyen de période $22 \mathrm{~h}$ pour les 3 poissons pour la sous-séquence de 960 heures qui présente, dans l'ensemble, la variance du profil $22 \mathrm{~h}$ la plus élevée. On peut observer des différences considérables entre les 3 poissons. Le premier et le deuxième présentent des profils en opposition de phase. La comparaison des valeurs chi-carré de la table et des profils de la fig. 2-A souligne à nouveau la variabilité inter-individuelle qu'il est difficile d'attribuer à l'interférence de deux systèmes oscillants, l'un étant le rythme circadien endogène et l'autre le cycle thermique imposé, puisqu'aucun rythme circadien endogène ne se manifeste durant les épisodes de conditions constantes. Cette variabilité intra-spécifique devrait donc s'expliquer à partir des différences inter-individuelles dans le système réactionnel des Poissons tant à l'égard des caractéristiques périodiques des signaux imposés (amplitude, phase, période et nature) que de leurs caractéristiques apériodiques (niveau). En outre, ceci ne tient pas compte de la possibilité d'effets consécutifs retardés, c'est-àdire de l'influence sur la régulation actuelle des conditions et des réponses antérieures, que le plan expérimental de cette recherche n'a pas pris en considération.

Septième série: Les conditions sont les mêmes que celles de la série précédente, sauf que le cycle thermique de cet épisode est fixé entre les limites de $17^{\circ} \mathrm{C}$ et $20^{\circ} \mathrm{C}$ (tableau 1,g; fig. 1,7).

On peut observer la réapparition d'un épisode périodique des réponses. A nouveau, le changement survenu dans les conditions imposées tend à produire un effet d'entraînement qui, comme dans les series précédentes, s'amortit progressivement.

Huitième série: Le niveau du cycle thermique est porté progressivement de $17^{\circ} \mathrm{C}-20^{\circ} \mathrm{C}$ à $27^{\circ} \mathrm{C}-30^{\circ} \mathrm{C}$ en une douzaine de jours (fig. 1,8). 

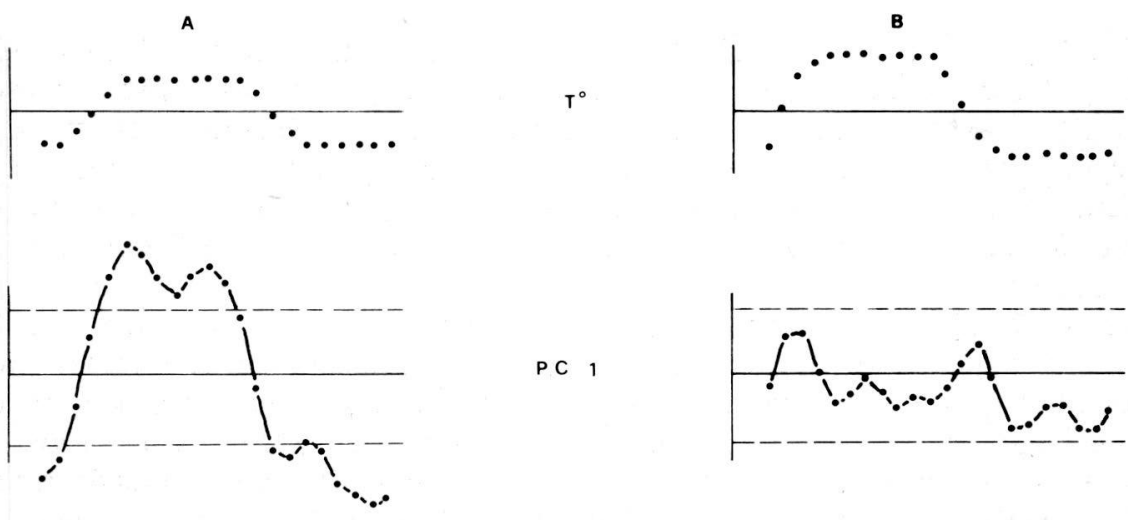

$T^{\circ}$
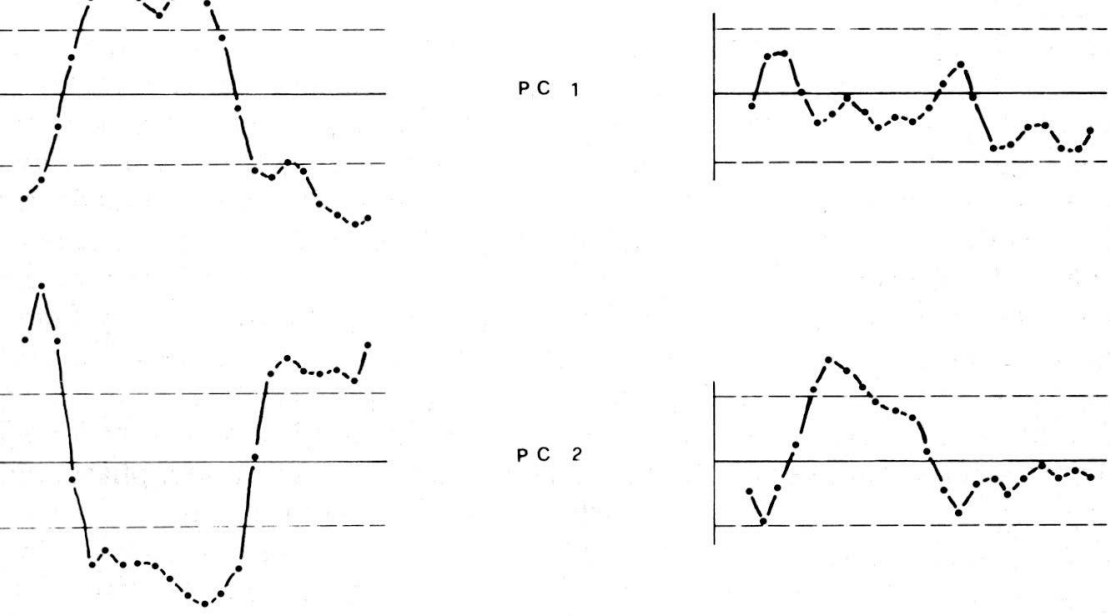

PC 2
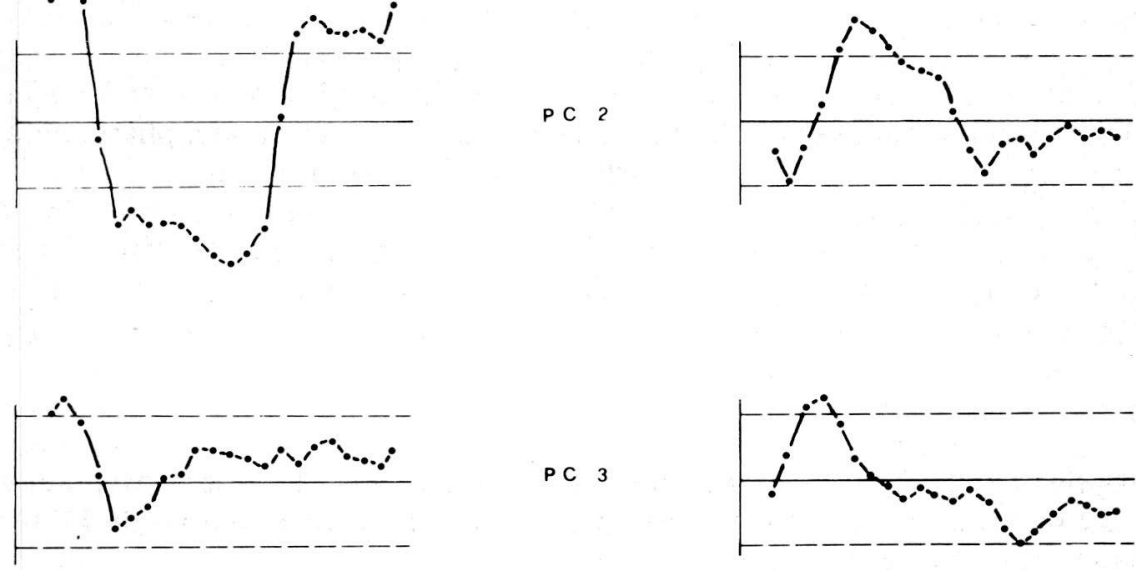

Fig. 2. - Exemple de profil moyen (chronogramme) des réponses de 3 Poissons pour deux sousséquences différentes. L'éclairement est constant (50 Lx).

En A, le profil moyen de 40 jours consécutifs dans lesquels une oscillation est observée et correspondant à la septième série de la fig. 1. En B, le profil moyen de 17 jours consécutifs d'un épisode comparable de la série 9. Le trait continu horizontal situe la valeur moyenne de la séquence, les deux traits discontinus situent les valeurs de +1 et de -1 écarts-type. En haut: profils des signaux thermiques. 


\author{
Neuvième série: Les conditions restent inchangées (LL $50 \mathrm{Lx}, \mathrm{t}^{\circ} 11 / 11,27^{\circ} \mathrm{C}$ \\ $-30^{\circ} \mathrm{C}$, fig. 1,9 ).
}

C'est au cours de cette neuvième série qu'un glissement de phase thermique a été opéré par la prolongation de $11 \mathrm{~h}$ de la fraction de température élevée du cycle. La figure 3 représente les ajustements de phase de l'activité pour la sous-séquence prenant cours à la fin de la huitième série (fig. 1.9).

La figure $2 \mathrm{~B}$ illustre à nouveau la variabilité inter-individuelle des ajustements de phase par le calcul du profil des 17 jours au cours desquels la réponse des Poissons est suffisamment périodique $(>1.2)$. Le glissement de phase est introduit le $19^{\circ}$ jour de la sous-séquence 9.

\title{
DISCUSSION ET CONCLUSION
}

Les résultats obtenus sur l'activité de l'Astyanax jordani présentent trois caractéristiques essentielles: 1) Les poissons ne présentent aucune composante circadienne endogène dans la régulation de leur activité, mais peuvent être entraînés par des signaux exogènes lumineux et thermiques; 2) la variabilité inter-individuelle est très marquée, en sorte que l'entraînement donne lieu à des réponses locomotrices parfois très divergentes d'un spécimen expérimental à l'autre. Il s'agit d'interpréter ces faits à la lumière des données relatives à la biologie générale des poissons cavernicoles, et de l'Astyanax jordani en particulier, telles qu'elles ont été exposées au début de ce travail. 3) Enfin, les rythmes induits par un cycle imposé tendent invariablement à s'amortir lorsqu'aucun changement ne vient affecter la stabilité du cycle. Tout se passe comme si un rythme effectif de l'activité ne pouvait apparaître qu'après un changement dans les caractéristiques du signal périodique et comme si l'absence d'une nouvelle modification aboutissait à l'amortissement complet du rythme des réponses (fig. 1). En outre, cette tendance à l'amortissement semble indépendante du niveau thermique qui, par ailleurs, est en relation avec le niveau de l'activité. Le comportement des poissons, analysé sur de très longues séries, s'apparente donc au phénomène de l'habituation, dans la mesure où un cycle lumineux ou thermique, non pertinent du point de vue de la biologie d'un cavernicole, peut être assimilé à une information périodique non renforcée.

L'absence d'une composante circadienne endogène de l'activité chez la forme cavernicole étudiée est conforme au tableau général qui se dégage des études effectuées sur les Téléostomes épigés qui ont été rappelées plus haut. Il n'est donc pas possible d'invoquer une explication de cet état de fait chez les cavernicoles à partir d'un mécanisme ancestral supposé plus parfait. En bref, les Poissons cavernicoles étudiés ne diffèrent en rien des formes épigées sous le rapport de la régulation temporelle, qu'il s'agisse de formes épigées actuelles ou d'hypothétiques formes ancestrales. Cette conclusion rejoint en outre celle de Husson (1971) à propos des cavernicoles en général. On se voit donc obligé de rechercher la signification biologique de ce fait dans une autre direction. 


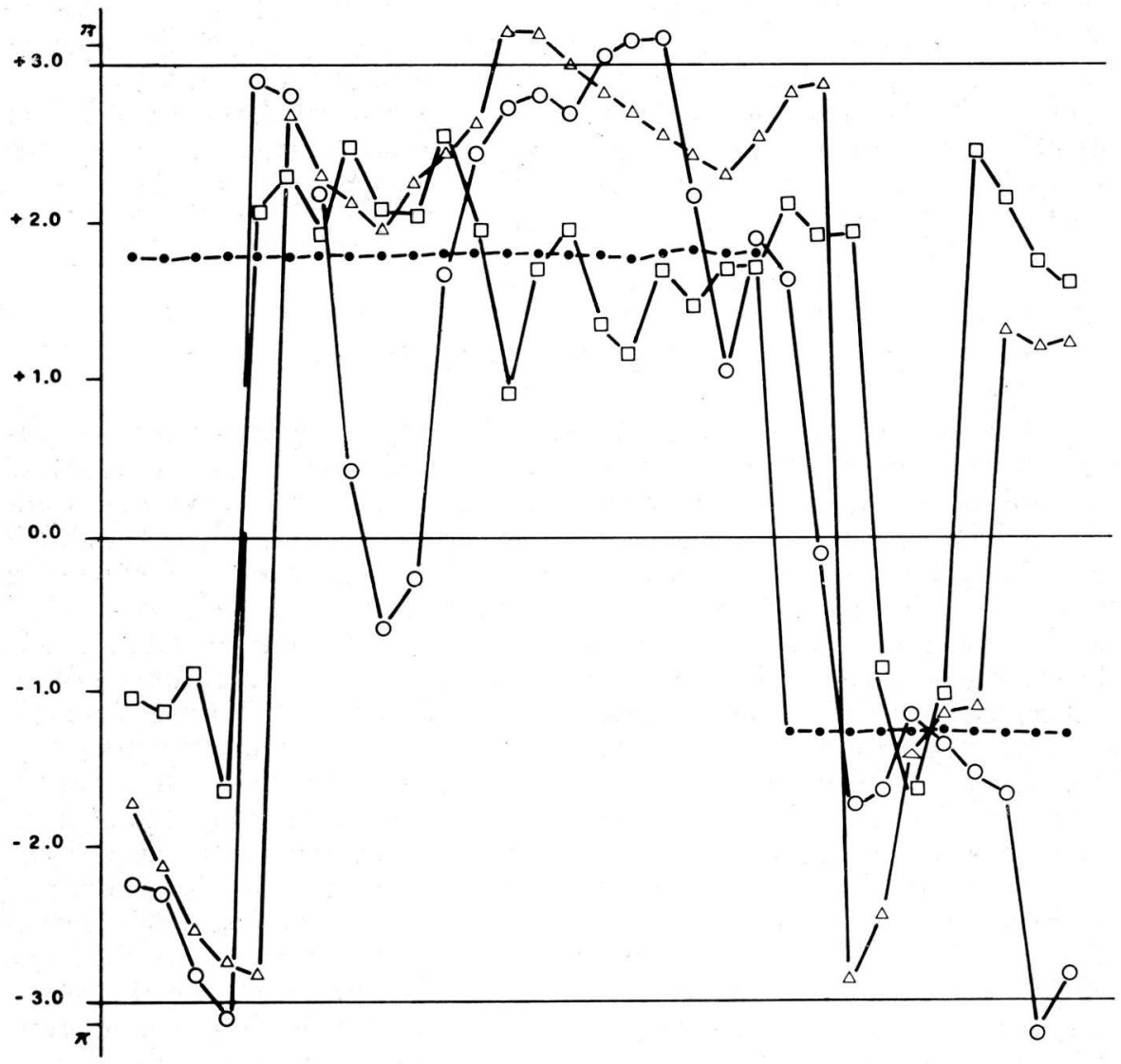

Fig. 3. - Exemple d'ajustement de phase des réponses des Poissons à la phase du signal thermique imposé.

La figure est obtenue en calculant la phase (par rapport au premier point de la séquence envisagée) d'une sinusoïde ajustée (moindres carrés) aux profils moyens de 22 heures calculés sur des sous-séquences de 110 heures avec décalage de $22 \mathrm{~h}$. L'angle de phase est exprimé en radian. La séquence correspond aux 28 premiers jours de la série 9 de la fig. 1 . La phase de la température est indiquée par les cercles noirs, celles des Poissons 1, 2 et 3 respectivement par un cercle, un carré et un triangle. Le glissement de phase du signal thermique $\left(180^{\circ}\right)$ est opéré le $19^{\circ}$ jour de cette séquence. 
Dans cette perspective, il n'est pas exclu de supposer que l'absence de régulation circadienne chez de nombreux Téléostomes ait constitué un caractère préadaptatif chez les formes ancestrales, tel qu'il est souligné par Kosswig (1962, 1963, 1965).

Dans ces cas comme dans celui du déficit de la régulation hygrométrique relevé de longue date par Fage (1931) à propos de certains Arachnomorphes, le milieu cavernicole apparaît comme un refuge électif susceptible d'être mis à profit dans le processus de peuplement souterrain (Vandel, 1964). Il faut néanmoins remarquer que l'absence de signaux périodiques exogènes de type nycthéméral qui caractérise les habitats souterrains définit une simple disponibilité et semble moins directement favorable, voire moins impératif pour la survie dans le cas des Poissons qu'un degré hygrométrique élevé et constant dans le cas des Arachnomorphes.

En ce qui concerne l'entraînement par un signal périodique exogène, tel que nos expériences le mettent en évidence, il est compatible avec les vues de Heuts (1951-1953) rappelées plus haut au sujet de l'incorporation passive des organismes cavernicoles aux variations faibles du milieu souterrain. Il faut toutefois préciser que nos résultats montrent un entraînement meilleur par les signaux thermiques que par les signaux lumineux (fig. 2 et 3 ). Si un tel processus d'adaptation passive est effectivement un trait majeur de l'adaptation des cavernicoles, il semble bien que la recherche d'un praeferendum thermique soit plus cruciale pour les Poissons. Ceci n'a du reste rien de surprenant, les réponses aux stimulus ${ }^{(1)}$ lumineux n'ayant une signification biologique de principe que pour permettre à un cavernicole de détecter les limites extrêmes de son biotope (Thinès, 1969) et n'intervenant en aucune façon à l'intérieur de ces limites, où les variations lumineuses sont inexistantes, au moins pour les troglobies des zones profondes. Les signaux thermiques, au contraire, peuvent contribuer à délimiter, à l'intérieur même du milieu aquatique souterrain, des zones différentielles très localisées, soit sous l'effet de courants, soit en raison de la proximité de parois ou de l'existence de dérivations résultant en la formation de gradients thermiques très particularisés. Comme il apparaît que le praeferendum thermique est variable d'un individu à l'autre mais est intraindividuellement stable, l'exploration variant en fonction du niveau imposé, on peut supposer que ce mécanisme contribue à disperser au maximum les individus d'une population sur l'ensemble du biotope aquatique souterrain dès qu'un seuil thermique local est atteint. L'utilité biologique de cet effet de dispersion est sans doute liée à la provende et au niveau saisonnier - à la reproduction. Cette interprétation trouve un argument supplémentaire dans le fait

(1) «Il a lieu de signaler que, dans une séance récente (24 janvier 1977), l’Académie des Sciences de Paris a rappelé que son Comité du langage scientifique a décidé depuis longtemps que les termes d'origine étrangère entrés dans la langue française devaient suivre, d'une façon générale, les règles de la grammaire française et non pas celles de leur grammaire d'origine; en conséquence elle précise que le pluriel de «stimulus» est «stimulus» et non «stimuli». 
que la tendance grégaire des Poissons cavernicoles est faible, voire nulle, l'orientation du corps dans les conditions naturelles étant très individualisée, comme Heuts et Leleup (1954) l'ont montré chez Caecobarbus. Des remarques similaires peuvent être faites à propos de l'Astyanax jordani (Thinès et Legrain, 1973).

En conclusion, les réponses locomotrices de cette dernière forme cavernicole à des signaux périodiques lumineux et thermiques révèle l'existence d'un praeferendum individualisé réglé prioritairement sur la température et dépourvu de composante endogène circadienne.

\section{RESUME}

L'activité locomotrice de Poissons cavernicoles adultes de l'espèce Astyanax jordani a été enregistrée dans l'isolement dans les conditions suivantes d'éclairement (LD:11/11 - 10 Lx - 100 Lx) et dans un cycle thermique $\left(11 / 11 ;-17 / 29 ; 19 / 22 ; 20 / 23\right.$ et $\left.27 / 30^{\circ} \mathrm{C}\right)$. Chacune des 7 séries longitudinales était étalée sur un minimum de 30 jours. Les résultats montrent: (1) qu'aucune régulation circadienne n'apparaît en conditions constantes; (2) qu'il existe un entraînement passif en LD (amplitude : $90 \mathrm{Lx}$ ) et dans des conditions thermiques périodiques (Amplitude $=3^{\circ} \mathrm{C}$ ). L'effet d'entraînement s'amortit et varie individuellement; (3) l'activité moyenne augmente avec la température; (4) l'ajustement de l'activité aux signaux périodiques est intra-individuellement stable. Ces résultats suggèrent que l'Astyanax jordani ne possède aucun oscillateur endogène de type circadien. L'adaptation thermique observée aurait les fonctions suivantes: (1) modifier le niveau d'activité en fonction du niveau de la température sous forme d'entraînement passif; (2) déterminer une activité exploratrice liée à la recherche d'un praeferendum thermique permettant aux Poissons de se maintenir à l'intérieur d'une zone bien délimitée du biotope souterrain en fonction de faibles variations locales de température.

ASCHOFF, J. 1965 - Circadian clocks. Amsterdam, North Holland Publ. Cy.

ANDREASSON, S. 1969 - Locomotory activity patterns of Cottus poecilopus Heckel. and C. gobio L. (Pisces). Oikos 20, 78-94.

ANDREASSON, S. 1973 - Seasonal changes in diel activity of Cottus poecilopus and C. gobio (Pisces) at the Arctic Circle. Oikos, 24, 16-23.

CITTA, M. 1977 - Time series analysis: the profilogram method. Int. J. Chronobiology, 4(3), 171-183.

FAGE, L. 1931 - Biospeologica LV. Aranae, 5e série précédée d'un essai sur l'évolution souterraine et son déterminisme. Arch. Zool Exp. Gén., 71, 99-291.

GIBSON, R.N. 1967 - Experiments on the tidal rhythm of Blennius pholis. J. Mar. Biol. Assoc. U.K. 47, 97-111.

HARRINGTON, R.W. 1963 - Twenty-four hour rhythms of interval self-fertilization and of oviposition by hermaphrodites of Rivulus marmoratus. Physiol. Zool., 36, 325-341.

HEUTS, M.J. 1951 - Ecology, variation and adaptation of the blind African cave fish Caecobarbus geertsi Blgr. Ann. Soc. Roy. Zool. Belg., 82, 2:155-230

HEUTS, M.J. 1953 - Regressive evolution in cave animals. Symp. Soc. Exp. Biol. VII. 290-309.

HEUTS, M.J. et LELEUP, N. 1954 - La géographie et l'écologie des grottes du Bas-Congo. Les habitats de Caecobarbus geertsi Blgr. Ann. Mus. Roy. Congo Belge, Tervuren, 35,71 pp.

HOFFMAN, K. 1969 - Die relative Wirksamkeit von Zeitgebern. Oecologia, 3, 184-206. 
HUSSON, R. 1971 - Rythmes biologiques et vie cavernicole. Bull. Soc. Zool. France 96, 301-316. KOSSWIG, K. 1962 - Über präadaptative Mechanismen in der Evolution vom Gesichtspunkt der Genetik. Zool. Anz., 169, 205-239.

KOSSWIG, K. 1965 - Génétique et évolution régressive. Rev. Quest. Scient., 136, 227-257.

LISSMANN, H.W. et SCHWASSMANN, H.O., 1965 - Activity rhythm of an electric fish, Gymnorhampichthys hypostomus Ellis. Z. Vergl. Physiol., 51, 153-171.

MEDIONI, J. 1967 - La distribution temporelle des activités animales et humaines. Paris, Masson, $189 \mathrm{pp}$.

MÜLLER, K. 1973 - Circadian rhythms of locomotor activity in aquatic organisms in the subarctic summer. Aquilo Ser. Zool., 14, 1-18.

MÜLLER, K. 1973 b - Seasonal phase and the duration of activity time in the Burbot Lota lota (L) (Pisces, Gadidae). J. Comp. Physiol., 84, 357-359.

PALMER, J.D. 1974 - Biological clocks in Marine Organisms. London, Wiley, 173 pp.

PARZEFALL, J. 1973 - Modifications du comportement de Poecilia sphenops (Pisces, Poeciliidae) consécutives à son passage à la vie cavernicole. Ann. Spéléol., 28, 283-290.

PETERS, G. 1973 - Caractères dégénératifs et constructifs chez une forme cavernicole phylogénétiquement récente de Poecilia sphenops (Pisces, Poeciliidae). Ann. Spéléol., 28, 301313.

PFEIFFER, W. 1963 - Alarm Substances. Experientia, 19, 113.

PFEIFFER, W. 1966 - Uber die Vererbung der schreckreaktion bei Astyanax (Characidae, Pisces). Z. Vererbungsl. 98, 97-105.

RENSING, L. 1972 - Periodic geophysical and biological signals as Zeitgeber and exogenus inducers in animal organism. Bioteorology, 5, suppl. 72, 113-124.

RICHARDSON, N.E. et McCLEAVE, J.D. 1974 - Locomotor activity rhythms of juvenile atlantic salmon (Salmo salar) in various light conditions. Biol. Bull. 147, 422-432.

SADOGLU, P. 1957 - Mendelian inheritance in the hybrids between the Mexican blind cave fishes and their overground ancestor. Verh. Dtsch. Zool. Ges. Graz., 1957, 432-439.

SAINT-GIRONS, M.C. 1971 - Le rythme circadien de l'activité des Mammifères. Les facteurs écologiques et éthologiques. Bull. Soc. Zool. France, 96, 331.

SCHEMMEL, C., 1973 - Les organes sensoriels cutanés du genre Astyanax (Pisces, Characidae) chez les formes occupant des biotopes souterrains. Ann. Spéléol., 28, 209-219.

SCHWASSMANN, H.O. 1971 - Biological rhythms. Dans: Hoar, N.S. et Randall, (Eds.) Fish Physiology, 1971, VI, 371-428.

SIEGMUND, R. et WOLFF, D.L. 1973 - Circadian Rhytmik und Grruppenverhalten bei Leucaspius delineatus (Pisces, Cyprinidae). Experientia, 29, 54.

STICKNEY, A.P. 1972 - The locomotor activity of juvenile herring (Clupea harengus harengus L.) in response to changes in illumination. Ecology 53, 438-445.

THINÈS, G. 1969 - L'évolution régressive des Poissons cavernicoles et abyssaux. Paris, Masson, $394 \mathrm{pp}$.

THINÈS, G. 1970 - Periodic variation in the photic behaviour of the Tench (Tinca tinca L.) in self-regulating conditions. J. Interdiscipl. Cycle Res., 1, 363-377.

THINÈS, G. et CAPON, D. 1975 - L'aveuglement expérimental de Poissons épigés et la cécité génétique des Poissons cavernicoles: effets sur le comportement alimentaire. Spelunca, Mémoires n. 8, 209-228.

THINES, G. et LEGRAIN, J.M. 1973 - Effets de la substance d'alarme sur le comportement des Poissons cavernicoles Anoptichthys jordani (Characidae) et Caecobarbus geertsi (Cyprinidae) Ann. Spéléol., 28, 291-297.

THINÈS, G. et PIQUEMAL, M. 1978 - Observations sur les comportements de Lucifuga subterranea Poey (Pisces, Ophidiidae), Poisson cavernicole de Cuba. (Intern. J. Spéléol., (in litteris). 
THINES, G., SOFFIÉ, M. et VANDENBUSSCHE, E. 1966 - Analyse du comportement alimentaire du Poisson cavernicole aveugle Anoptichthys Gen. et d'hybrides et Caecobarbus geertsi Blgr. Intern. J. Speleol., 4, 139-169.

THINÈ, G., WOLFF, F., BOUCQUEY, C. et SOFFIE, M. 1966 - Etude comparative de l'activité du Poisson cavernicole Anoptichthys antrobius Alvarez et de son ancêtre épigé Astyanax mexicanus (Philippe). Ann. Soc. Roy. Zool. Belg., 96, 61-115.

VANDEL, A. 1964 - Biospéléologie. Paris, Gauthier-Villars, 619 pp.

VARANELLI, C.C. et McLEAVE, J.D. 1974 - Locomotor activity of Atlantic Salmon parr (Salmo salar L.) in various light conditions and in weak magnetic fields. Anim. Behav., 22, $178-186$.

WEYERS, M. 1974 - L'effet de signaux périodiques d'éclairement et de température sur les régulations circadiennes du comportement de Poissons d'eau douce (Thèse Université de Louvain), 192 pp.

WILKENS, H. 1973 - Ancienneté phylogénique et degrés de réduction chez les animaux cavernicoles. Ann. Spéléol., 28, 327-330.

WINFREE, A.T. 1974 - Suppressing Drosophila circadian rhythm with dim light. Science, 183, 970-972. 